\title{
SIMETRÍA LOCAL, ORDENAMIENTO MAGNÉTICO Y PROCESO DE DESORCIÓN DE OXÍGENO EN EL SISTEMA (LA1- XGDX)1,85SR0,15CUO4
}

D. R. Sánchez 1, M. Márquez Jácome 2, S. García 3 y E. Baggio-Saitovitch 1

SUMILLA: El sistema (La1-xGdx)1,85Sr0,15CuO4 ( ) dopado con 1\% at. de 57Fe fue estudiado por espectroscopia Mössbauer del 57Fe, susceptibilidad AC y difracción de rayos X. Los estudios a temperatura ambiente nos permitieron interpretar las diferentes especies de $\mathrm{Fe}(\mathrm{Cu})$ en función de la vecindad local de oxígeno y las características de las fases $\mathrm{T}(\mathrm{x}=0)$, $\mathrm{T}^{*}(\mathrm{x}=0,45)$ y T' $(\mathrm{x}=1)$. Las medidas a 4,2 K mostraron que el tipo de orden magnético del $\mathrm{Fe}(\mathrm{Cu})$ es influenciado por la geometría local de oxígeno. Se efectuaron mediciones Mössbauer in situ a temperaturas altas en el compuesto Gd1,85Sr0,15CuO4 (fase T') a una presión reducida. La temperatura de Debye obtenida para el Fe en este compuesto es aproximadamente $328 \mathrm{~K}$. El análisis de la variación de las intensidades relativas de los diferentes subespectros permitió monitorar la desorción de los oxígenos cercanos a los iones de Fe.

ABSTRACT: The (La1-xGdx)1,85Sr0,15CuO4 ( ) system doped with 57Fe (1 at \%) was studied by 57Fe Mössbauer spectroscopy, AC susceptibility and x-ray diffraction. An interpretation of the different sites of $\mathrm{Fe}(\mathrm{Cu})$ as a function of the neighboring oxygen and the characteristics of the phases $T(x=0), T^{*}(x=0,45)$ and $T^{\prime}(x=1)$, was obtained at room temperature. The 4,2 K spectra show that the magnetic ordering of the $\mathrm{Fe}(\mathrm{Cu})$ is influenced by the oxygen local geometry.In situ high temperature Mössbauer measurements were performed on a Gd1,85Sr0,15CuO4 sample ( $\mathrm{T}^{\prime}$ phase) at reduced pressure. A Debye temperature of about $328 \mathrm{~K}$ was obtained and the analysis of the variation of relative intensities of the various subspectra allowed to monitor the oxygen desorption near the Fe ions.

\section{INTRODUCCIÓN}

Las cerámicas superconductoras de alta temperatura crítica, descubiertas en Diciembre de 1986 [1], continúan siendo hoy en día uno de los temas más investigados en el área de la ciencia de los materiales. El número de publicaciones es numerosa, resaltando un carácter claramente aplicativo, incluyendo modelos teóricos sobre los mecanismos que estable-cen el estado superconductor.

Se han propuesto varios modelos teóricos con la finalidad de describir la naturaleza del estado superconductor en las diferentes familias de compues-tos cerámicos conocidos. Sin embargo, en general, dichos modelos tienen sólo un éxito parcial cuando intentan describir las propiedades en su conjunto. En este momento el estado de la teoría es tal que no es posible predecir con certeza nuevos compuestos superconductores. En particular, resulta sorprendente que varios cupratos bidimensionales que tienen una gran analogía estructural con los óxidos supercon-ductores conocidos, no lo son en absoluto, siendo incluso dieléctricos con orden magnético. Por esta y otras razones, el estudio de las contrapartes no superconductoras ha venido recibiendo recientemente mayor atención, con la finalidad de determinar el papel que desempeña cada detalle estructural en las propiedades de estos compuestos.

Los detalles estructurales relacionados a la vecindad local de oxígenos en el sitio del cobre, o vacancias, son relevantes para el establecimiento del estado superconductor, puesto que influyen intensamente en la densidad de los portadores de carga, propiedades magnéticas y en los mecanismos de conducción. En particular, un desorden en los planos Cu-O2 de los cupratos laminares es determinante, puesto que puede actuar como un mecanismo de ruptura de los pares de Cooper [2]. Por lo tanto, es de gran interés obtener información directa sobre la configuración local en el sitio del cobre para correlacionarlas con las medidas macroscópicas.

Resumiendo lo dicho anteriormente es entonces de gran interés actual establecer una correlación entre los aspectos estructurales de los cupratos bidimen-sionales y sus propiedades. Una forma conveniente de realizar esta investigación es caracterizar un conjunto de compuestos (un sistema) mediante una técnica microscópica que proporcione información local a nivel de sitios cristalográficos y establecer una correspondencia con las propiedades macroscó-picas como son, por ejemplo, mediciones de parámetros de transporte y parámetros magnéticos. Precisamente en este trabajo usamos este enfoque y utilizamos como técnica microscópica la Espectros-copía Mössbauer del 57Fe en régimen de transmi-sión; dicho isótopo juega el papel de núcleo sonda en las estructuras cristalográficas. También nos permite obtener información acerca de la dependen-cia de la susceptibilidad magnética con la tempe-ratura.

Se escogió el sistema (La1-xGdx)1,85Sr0,15CuO4 por las siguientes razones:

1. Representa el caso más simple de configura-ciones en torno al átomo de cobre puesto que existe sólo un tipo de vecindad de simetría octaedral, piramidal y cuadrática planar por celda unitaria.

2. Ofrece la posibilidad de variar las concentraciones de los iones alcalino-terrosos, lo que permite, además, obtener una información más amplia y al mismo tiempo disponer de un sistema en el cual se varíe de manera controlada y sistemática los defectos asociados con los dopantes.

3. Permite pasar gradualmente, a través de la sustitución del La por Gd, de la fase T super-conductora original a las fases no supercon-ductoras $\mathrm{T}^{*}$ y T' (Figura1); estas últimas están estrechamente relacionadas con la primera debido a la pérdida de 1 y 2 átomos de oxígeno respectivamente, lo que representa una fuente adicional de información valiosa [3].

4. La fase $\mathrm{T}^{\prime}$ es isomorfa con los compuestos de la serie Nd2-xCexCuO4-d, que son los únicos superconductores de tipo n en el 
estado normal [4], motivo por el cual son particularmente interesantes.



5. Los efectos de las diferentes presiones parciales del oxígeno durante el proceso de obtención se reflejan isotrópicamente en el material al haber un sólo tipo de sitio para el cobre.

El uso del 57Fe como núcleo Mössbauer tiene la ventaja de proporcionar información directa acerca de los cambios que se producen en los planos de $\mathrm{CuO}$ [5-11] ya que el Fe3+ sustituye al Cu2+. No obstante, debe tenerse un especial cuidado en las interpretaciones de los resultados experimentales ya que el Fe tiene tendencia a retener átomos de oxígenos adicionales, situación que no ocurre con los átomos de $\mathrm{Cu}$. Además, se sabe que el átomo de Fe tiene momento magnético y su presencia en el compuesto puede inducir un ordenamiento mag-nético de largo alcance involucrando adicionalmente los momentos de otros átomos del compuesto.

En este trabajo se estudian los cambios estructurales en el sistema (La1-xGdx)1,85Sr0,15CuO4 por espectros-copía Mössbauer y susceptibilidad magnética. Uno de los objetivos es lograr, a través de la interpre-tación sistemática del conjunto de espectros, una identificación segura de las diferentes configuracio-

nes de oxígeno entorno de los átomos de cobre y de sus densidades relativas, lo cual será utilizado en estudios posteriores correlacionando -por ejemplo- la densidad de defectos con las propiedades macros-cópicas. Un segundo objetivo es obtener informa-ción sobre la influencia de la geometría local en el tipo de orden magnético existente (este orden fue observado por espectroscopía Mössbauer en el sitio del Cu). Finalmente, nos concentramos en el estudio de la fase T', que como mencionamos inicialmente, es isomorfa con los superconductores tipo n. Obtendremos información sobre la modificación de la vecindad local del oxígeno durante el proceso de desorción de oxígeno inducido por el tratamiento térmico al vacío.

No se disponen publicaciones previas de estudios en las fases $\mathrm{T}^{*} \mathrm{y} \mathrm{T}^{\prime}$ utilizando el núcleo resonante justamente en los planos CuO. Hay un estudio realizado a temperatura ambiente en estas muestras [12,13], siendo ésta nuestra segunda publicación en estos cupratos bidimensionales. Existe una publica-ción de 1993 utilizando el isótopo Mössbauer 155Gd[14] fuera de los planos anteriormente mencio-nados.

\section{EXPERIMENTAL}

Se mezclaron cantidades apropiadas de La2O3, SrCO3, Gd2O3 de alta pureza y 57Fe2O3 enriquecido en proporciones estequiométricas y luego empasti-lladas. Se realizaron dos tratamientos térmicos previos en aire: a 900oC por 12 horas y 980 oC por 15 horas respectivamente; el tratamiento térmico final fue realizado a 1100 oC en un flujo de oxígeno por 15 horas. Se efectuaron también operaciones de trituración, molido y empastillamiento antes de cada tratamiento térmico. En cada etapa las muestras fueron enfriadas a razón de $450 \mathrm{C} / \mathrm{h}$.

Todos los espectros Mössbauer fueron tomados con una fuente de 57Co:Rh moviéndose sinusoidalmente. Para efectuar las medidas Mössbauer a 4,2 K, tanto la fuente como la muestra se sumergieron en He líquido dentro de un crióstato Sulfrian Cryogenics. Para las medidas por encima de $300 \mathrm{~K}$ la fuente fue mantenida a temperatura ambiente.

Las medidas de susceptibilidad AC fueron realiza-das utilizando un susceptómetro construido en el CBPF y con un campo de 1 Oe. Los padrones de difracción de rayos X reproducen las características estructurales reportadas para las muestras no dopadas3. Como se esperaba, la substitución de 1 a 3\% de los átomos de Cu por Fe no induce ningún cambio en la estructura cristalina.

\section{SIMETRÍA LOCAL EN EL SISTEMA (La1xGdx)1,85Sr0,15CuO4}

Se estudó la serie de muestras del sistema (La1-x Gdx)1,85 Sr0,15CuO4 ( ), donde el 1\% de sus átomos de Cu fueron substituidos por átomos de Fe, por espectroscopia Mössbauer y difracción de rayos X. El análisis global de los datos nos permite obtener una interpretación de las diferentes especies de Fe en términos de la vecindad local, como carac-terística de las fases T, T* y T'.

Comenzaremos exponiendo los resultados del análisis por rayos X para todas las muestras. Esto nos permite, entre otras cosas, conocer la calidad y la pureza cristalográfica de las muestras utilizadas. La figura2 grafica los espectros de rayos X de las muestras pertenecientes a las fases $\mathrm{T}, \mathrm{T}^{*}$ y $\mathrm{T}^{\prime}$, dopadas con $1 \%$ de Fe. Todos los padrones presen-tan picos bien definidos, indicando una buena cristalización y pureza. Los padrones de difracción fueron indexados con una estructura perovskita tetragonal.

En la figura 3 se presenta la variación de los parámetros de la red en función de la concentración de Gd. Se puede observar que el 
parámetro a crece monótonamente y el parámetro c disminuye lineal-mente siguiendo el orden de las fases T®T*ßT'. También la relación c/a y el volumen $\mathrm{V}$ disminuyen en el mismo orden. La disminución de la relación c/a indica una disminución gradual de la distorsión Jahn Teller (el cual es muy fuerte para los iones de Cu2+ en la coordinación octahedral) y disminuye también en el orden $\mathrm{T}^{\circledR} \mathrm{T}^{*}{ }^{\circledR} \mathrm{T}^{\prime}$, resultado que está de acuerdo con los datos de la literatura [3].



Figura 2. Padrones de difracción de rayos-x de la serie (La1xGdx)1,85Sr0,15CuO4, dopados con $1 \%$ de Fe.
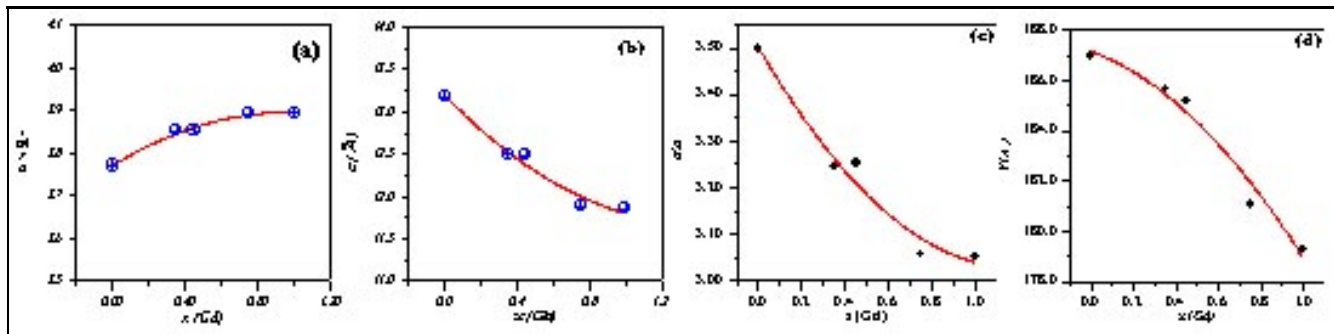

Figura 3. Parámetros de red de la serie (La1-xGdx)1,85Sr0,15CuO4 en función de la concentración de Gd.(a) parámetro a, (b) parámetro c, (c) relación c/a, (d) Volumen V.

Los espectros Mössbauer típicos para las muestras de la serie (La1-xGdx)1,85Sr0,15CuO4 con x=0,00, 0,35, 0,45, 0,75, 1,00, dopados con $1 \%$ de Fe se muestran en la figura 4. Para analizar todos los espectros de las fases $\mathrm{T}, \mathrm{T}^{*}$ y $\mathrm{T}^{\prime}$, se consideraron cinco especies de Fe con diferentes valores de desdoblamiento quadrupolar, , a los cuales hemos denominado espe-cies A, B, C, D y E. Se puede notar que las áreas relativas y los valores de , de los respectivos subespectros, cambian con la concentración de Gd.

En la figura 5 se muestra la dependencia del desdoblamiento cuadrupolar con la concentración de Gd. Se nota una ligera disminución de los valores de para las especies A, B y C. Mientras que los valores de corrimiento isomérico (CI) se mantienen casi constantes y corresponden al estado de alto spin ( ) del Fe3+. Esta disminución de los valores de está relacionado a la variación regular de los parámetros de red inducido por la sustitución del átomo La por Gd. Este hecho esta también relacionado con la aparición de nuevas vecindades de oxígenos que relajan la red.

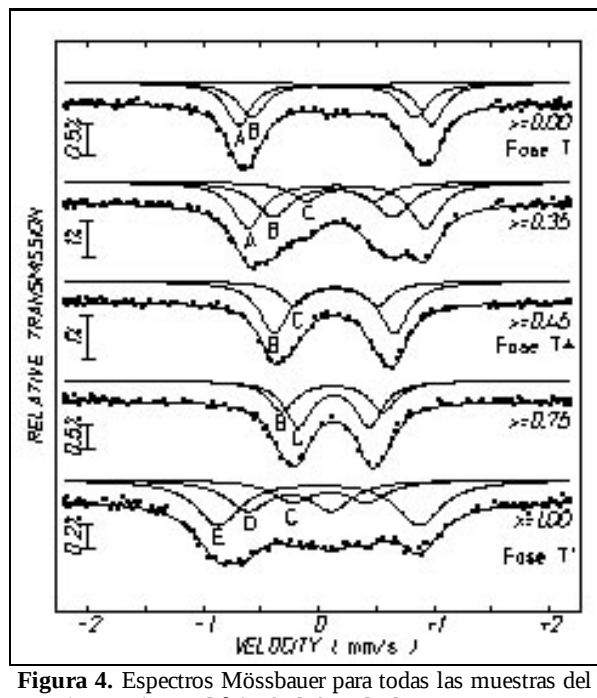

sistema (La1-xGdx)1,85Sr0,15CuO4 a temperatura

ambiente. 


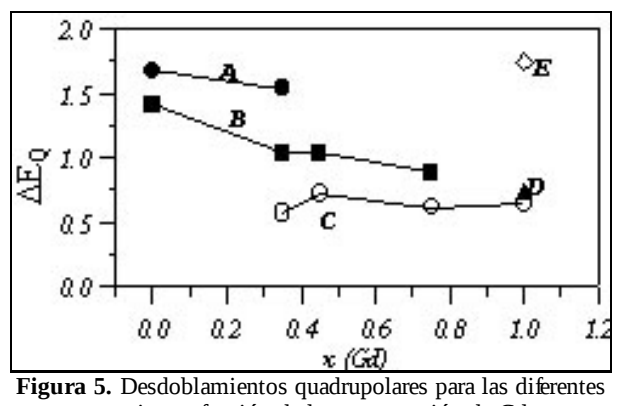

especies en función de la concentración de Gd.

En las muestras de la fase T' ocurre un notable cambio: aparecen dos nuevas contribuciones D y E, mostrando valores reducidos de CI. La especie D tiene un CI negativo de $-0,11 \mathrm{~mm} / \mathrm{s}$ en relación al fierro metálico, el cual es muy similar de aquel obtenido para una de las especies de Fe localizadas en las cadenas del YbaCuO [8-10]. A ninguna de estas contribuciones se le puede atribuir el estado de alto spin del Fe3+. Esto probablemente correspon-de a una valencia mayor y/o a un estado de spin intermedio o bajo.

En la figura 6 se muestra la dependencia de las áreas relativas versus el contenido de Gd, para las diferen-tes contribuciones. En este análisis adicionamos las áreas de las especies A y B, asumiendo que ellas están relacionadas con la coordinación octaedral. La especie B es atribuida a la existencia de defectos cerca del sitio del $\mathrm{Cu}(\mathrm{Fe})$ los cuales son intrínsecos al compuesto La2CuO4. Estos defectos son también características específicas de la estructura del K2NiF4, tales como vacancias e intersticios de oxíge-nos [11].

El doblete asociado a la especie A, con un mayor que el correspondiente a la especie B, está relacionado a las posiciones de la red que no están afectadas por defectos estructurales próximos. Obsérvese también que la especie B está presente en la fase T*, aún cuando los padrones de difracción corresponden a la fase $\mathrm{T}^{*}$ pura, la cual, como recor-damos, tiene solamente coordinación piramidal en una red ideal perfecta. El hecho de que la especie B permanezca en la fase T* se debe a la capacidad de los iones de Fe de atraer átomos de oxígenos extras en torno de su vecindad.

El área correspondiente a la especie $C$ tiene un máximo en torno de $\mathrm{x}=0,75$; por esa razón esta especie fue atribuida a la fase $\mathrm{T}^{*}$ (coordinación piramidal de oxígenos). El hecho de que su máximo no corresponda a $\mathrm{x}=0,45$ (como era de esperar) puede ser debido a la capacidad del Fe de atraer oxígenos extras. La contribución de los sitios (A, B) del compuesto no dopado con Gd, correspondientes a un octaedro sin defectos y un octaedro afectado por defectos, decrece sistemáticamente con la concentración de Gd, y por lo tanto también sus correspondientes . La especie B permanece en la muestra hasta al menos $\mathrm{x}=0,75$.

Teniendo en cuenta que los resultados observados en la fase T' representa un cambio inesperado de la variación regular exhibida previamente y con la finalidad de efectuar un estudio más preciso de esta fase, se preparó un conjunto adicional de muestras cambiando el contenido de Gd dentro del rango estrecho para el cual la fase T' permanece como tal. En todos estos casos los espectros pueden ser analizados completamente en términos de las contribuciones C, D y E, con proporciones diferen-tes de sus áreas relativas [12].

En la figura 7 se muestra la variación de las correspondientes áreas de los dobletes C, D y E (para las muestras sintetizadas en aire a 1050oC) versus concentración de Gd. Esto permite proporcionar mayor evidencia experimental de nuestras interpretaciones. Es necesario una previa consideración estructural para explicar estos resul-tados: ya que los iones de Gd3+ y Sr2+ tienen radios iónicos diferentes, puede ser factible promover un orden de acomodación de corto alcance, induciendo diferentes vecindades locales. Müther [14] reciente-mente ha publicado un estudio Mössbauer con 155Gd, indicando que los iones de Gd, en el compuesto Gd1,85Sr0,15CuO4 (fase T'), están localizados principalmente en dos sitios no equiva-lentes: uno de ellos con Sr2+ en los sitios catiónicos más cercanos, y el otro en un lugar con fuertes distorsiones locales, causados por dos vecinos de Sr2+, probablemente asociado con un defecto de oxígeno.

Los resultados - resumidos en la figura 7 - están de acuerdo con esta idea. Puede observarse también que cuando el contenido de Gd se incrementa, la intensidad del doblete C (el cual está asociado a la configuración más oxigenada para el Fe presente en la fase $\mathrm{T}^{\prime}$ ) disminuye, mientras que la contribución del doblete E aumenta. El área relativa de la especie D se mantiene casi constante.

Todo parece indicar que el sistema está involucrado en un proceso donde la pérdida regular del oxígeno se debe al dopaje de Gd, apareciendo al mismo tiem-po vacancias debido al proceso de acomodación de los átomos en la red. De este modo, el proceso puede ser descrito por una transición $\mathrm{C} \circledast \mathrm{D} \circledast \mathrm{E}$, sugi-riendo que la especie D corresponde a una coordi-nación cuadrada, mientras que la especie E está relacionada a alguna vecindad planar distorsionada, probablemente asociada a una vacancia de oxígeno. Debemos destacar también el hecho que las muestras preparadas en oxígeno fueron siempre más ricas en las especies D que las correspondientes preparadas en aire.

En conexión con el superconductor de tipo n (estructura T'), para el cual se ha demostrado que el papel de los defectos es relevante para el estableci-miento del estado superconductor, estos resultados apuntan al hecho de que los defectos son intrínsecos a la fase $\mathrm{T}^{\prime}[13]$.

\section{ORDENAMIENTO MAGNETICO LOCAL EN EL SISTEMA (La1-xGdx)1,85Sr0,15CuO4}

En los cupratos superconductores, los detalles es-tructurales, tales como el contenido local de oxígeno o las coordinaciones locales, son considerados determinantes para sus propiedades macroscópicas. Nos concentraremos en la influencia de la configuración de oxígeno sobre el tipo de orden magnético en los compuestos mencionados (La1-xGdx)1,85Sr0,15CuO4. Este orden ha sido probado por espectroscopia Mössbauer del 57Fe en los sitios del cobre. Pese a la perturbación local que podría posi- 
blemente ser inducida por el substituyente Fe, los campos magnéticos hiperfinos encontrados son típi-cos para las tres fases [15]. La figura 8 muestra los espectros Mössbauer de la muestra La1,85Sr0,15CuO4 (fase T) dopado con 3 \% de Fe a 300, 110 y 4,2 K de temperatura respectiva-mente. En la figura 8 se observa la presencia de una considerable distribución de campos hiperfinos en el espectro a 4,2 K de temperatura; ésta es una característica común en la mayoría de los sistemas spin - glass. La asimetría observada resulta de una reorientación del campo hiperfino Bhf relativo al eje principal del gradiente de campo eléctrico Vzz, el cual esta orientado en la dirección c. El ángulo de inclinación q del Bhf respecto de Vzz es de 52o, en lugar del valor de 90o, el cual es observado en el compuesto La2CuO4 [9]. Este ángulo también es consistente con una orientación al azar del campo hiperfino Bhf relativo a Vzz. Como la dirección Vzz relativo al eje c no cambia, este resultado muestra que el momento magnético del Fe3+ no está en el plano Cu-O sino que está inclinado en la dirección del eje c. Estos datos están de acuerdo con los resultados obtenidos por Imbert[16] para la región superconductora del sistema $\mathrm{La2-xSrxCuO4} \mathrm{y} \mathrm{es} \mathrm{inter-pretado} \mathrm{como} \mathrm{sigue:} \mathrm{el} \mathrm{desdoblamiento} \mathrm{magnético}$ hiperfino no refleja el ordenamiento magnético espontáneo de la subred del Cu. Este nuevo tipo de estructura magnética hiperfina es atribuida a un congelamiento del spin local, el cual es inducido por las impurezas de Fe.

En la figura 9 a se muestra las medidas de susceptibilidad AC versus la temperatura para la muestra (La0,55Gd0,45)1,85Sr0,15CuO4 (fase $\mathrm{T}^{*}$ ) dopada

con 1, 2 y $3 \%$ de Fe respectivamente. No se observa orden magnético en estas muestras, pero si exhiben paramagnetismo con un momento magnético cons-tante para el Gd. El momento magnético efectivo peff es del orden de 6,7 mB/átomo que es típico del estado fundamental de Gd3+ y de acuerdo con la regla de Hund. El valor de la temperatura de Curie-Weiss es de -7,6 K. El valor negativo es una señal de la existencia de una interacción antiferromagnética entre los iones de Gd.

En la figura $9 \mathrm{~b}$ se grafican los espectros Mössbauer de la muestra (La0,55Gd0,45)1,85Sr0,15CuO4 (fase T*) dopada con 3\% de Fe a las temperaturas que se indican. Se observa un sexteto magnético hiperfino y cuadrupolar en el espectro Mössbauer a 4,2 K de temperatura, indicando un orden magnético de largo alcance. El espectro fue ajustado con dos sextetos hiperfinos, los cuales están relacionados con las dos especies de Fe observados a temperatura ambiente: uno atribuido a una coordinación octaedral para el Cu (Fe) y la otra a una coordinación piramidal. El ángulo entre Vzz y el campo magnético hiperfino Bhf encontrado en esta fase es del orden de 70o, demostrando que el momento magnético del Fe3+ no está en el plano Cu-O sino inclinado en la dirección del eje c. Ya que no se observa un ordenamiento magnético definido de la subred Cu-O en la magnetización AC, se puede asumir que el Fe está induciendo un ordenamiento magnético local, el cual es visto como un campo hiperfino estático en el espectro Mössbauer.

Las medidas de susceptibilidad AC para las muestras de la fase T' (Gd01,85Sr0,15CuO4, dopadas con 1,2 y 3 \% de Fe) y mostradas en la Figura 10a, exhiben órdenes magnéticas interesantes. Existen dos transiciones magnéticas a aproximadamente $260 \mathrm{~K}$ y $20 \mathrm{~K}$ de temperatura. La transición a la temperatura TN=260K está indudablemente asociada con el ordenamiento antiferromagnético de la subred $\mathrm{Cu}-\mathrm{O} 3$. Por otro lado, la naturaleza de la transición magnética a baja temperatura no está asociada con el ordenamiento antiferromagnético del Gd, pero si lo está a las interacciones $\mathrm{Gd}-\mathrm{Cu}, \mathrm{Cu}-\mathrm{Cu}$ y otras.

En la figura $10 \mathrm{~b}$ se exhibe los espectros Mössbauer para la muestra Gd01,85Sr0,15CuO4 (fase T') dopada con 3\% de Fe, a las temperaturas que se indican. Basados en los estudios Mössbauer de Fe en el compuesto La2CuO4, deberíamos esperar un espectro magnéticamente desdoblado a la temperatura de $\mathrm{T}=110 \mathrm{~K}$ puesto que, por debajo de $\mathrm{TN}=260 \mathrm{~K}$ hay un estado de Neel, el cual está relacionado con el ordenamiento antiferromagnético de la subred del Cu-O; los momentos magnéticos del Fe deberían seguir el mismo ordenamiento magnético del $\mathrm{Cu}$. Los espectros Mössbauer, sin embargo, reproducen los resultados obtenidos a temperatura ambiente. Se observa también a la temperatura de 4,2K que no hay líneas magnéticas en el espectro, sino una única línea ensanchada, típica de un efecto de relajación magnética.

En este caso la agitación térmica permite la precesión rápida de los momentos del Fe y de este modo también del campo hiperfino del Fe, conduciendo a un colapso del espectro magnético Mössbauer. Así mismo, la ausencia del ensancha-miento de relajación magnética en el espectro a la temperatura de $110 \mathrm{~K}$ muestra que las fluctuaciones del spin son muy rápidas e imposibles de ser detectadas por espectroscopia Mössbauer. Mayor información sobre este punto puede ser obtenida a partir del ajuste apropiado de los espectros medidos en función de la temperatura y campo magnético aplicado [17].

\section{DESORCIÓN DE OXIGENO EN LA FASET".}

Como mencionamos inicialmente, los detalles estructurales relacionados a la vecindad local de oxígeno (coordinación y vacancias) son relevantes para las propiedades de los óxidos superconduc-tores. Entre los óxidos del tipo TR2CuO4 (TR=tierra rara), los cuales cristalizan en la fase $\mathrm{T}^{\prime}$ (coordina-ción cuadrada planar de oxígeno) son particularmente interesantes puesto que tienen la misma estructura de los superconductores tipo n (Nd01,85Ce0,15CuO4) [4].

En la preparación de las muestras del compuesto Gd01,85Sr0,15CuO4 (fase T'), substituimos de 1 a 3\% de los átomos de Cu por 57Fe, con la finalidad de estudiar la modificación de la vecindad local de oxígeno durante el proceso de desorción inducido por el tratamiento térmico en vacío. La información de las medidas Mössbauer in situ fueron limitadas a $250{ }^{\circ} \mathrm{C}$, puesto que a una temperatura más alta se produce la descomposición de la muestra.

De los resultados experimentales se obtiene la dependencia del área de absorción versus la temperatura, proporcionando información acerca de la dinámica de la red (temperatura de Debye) de los iones de Fe en estos compuestos. El análisis de las intensidades relativas de los subespectros nos permite monitorear la desorción de oxígeno cerca de los iones de Fe en función de la temperatura y de la presión residual.

Las muestras fueron mantenidas en un horno de temperatura controlada, con portamuestras de nitrato de boro y ventanas de mylar, lo cual nos permite realizar estudios in situ a temperaturas altas y bajo ciertas condiciones de vacío. La fuente de Co:Rh se mantuvo a temperatura ambiente y los tiempos de recolección de los datos fueron del orden de 12 horas a temperatura fija. 
La figura 11 grafica los espectros Mössbauer de la muestra de la fase T', dopada con 3\% de Fe y efectuada en un vacío de 10-5 atm a 400 C. Se observa inicialmente una disminución gradual de la magnitud de los desdoblamientos cuadrupolares (dobletes); sin embargo, la información principal obtenida está asociada con la población de los dobletes asociados a las especies D y E (figura 12). La descomposición de los espectros a temperatura ambiente (figuras 11 y 12) indican una clara modificación de la estructura de la muestra vistos a través de los parámetros hiperfinos, ya que estos cambian drásticamente después del calentamiento. Se observa que a la temperatura de $350 \mathrm{C}$ la muestra comienza a descomponerse; por esta razón nuestro estudio se limitó a calentar las muestra hasta la temperatura de $280 \mathrm{C}$.

En la figura 13 se grafican los espectros Mössbauer obtenidos para la muestra con 3\% de Fe, calentada hasta 250 C en un vacío de 10-2 atm. En este caso también la información principal obtenida está ligada con la población de las especies D y E, las cuales son graficadas en las figuras 14a y 14b. La población de la especie C no ha sido mostrada ya que se mantiene constante con el incremento de la temperatura.

Es importante notar que las poblaciones asociadas a los dobletes D y E no son recuperadas cuando la muestra es enfriada nuevamente hasta la temperatura ambiente. En las figuras 14a y 14b se observa la variación de las poblaciones asociadas con las especies D y E que siguen en el proceso de calenta-miento y enfriamiento (conforme indican las flechas). Nótese que la principal pérdida de oxígeno en torno del Fe tiene lugar por encima de los 200 oC de temperatura, lo que está asociada además con la difusión de oxígeno en esos sistemas. Además se observa que la especie E se forma a costa de la especie D, sugiriendo que esta última tiene más oxígenos en su vecindad. Estas dos vecindades locales pueden ser intrínsecas en la fase $\mathrm{T}^{\prime}$. La especie $\mathrm{D}$ puede corresponder a una coordinación planar cuadrada, típica del $\mathrm{Cu}$ en esta fase, mientras que la especie E puede estar relacionada a alguna vecindad planar cuadrada distorsionada asociada a una vacancia de oxígeno $[8,16]$. La configuración atribuida a la especie D puede transformarse en la especie E, si un oxígeno fuera retirado de una especie de coordinación cuadrada. Este proceso necesita una cierta energía de activación y puede ser irreversible. Estas dos vecindades locales pueden ser intrínsecas a la fase T'.

El área de absorción medida en un experimento Mössbauer a diferentes temperaturas T nos proporciona información sobre el desplazamiento medio cuadrático de los isótopos Mössbauer en el absorbedor. Dentro del modelo de Debye y a elevadas temperaturas ( ) el factor $\mathrm{f}$ puede ser aproximado por [18]

$$
\operatorname{Ln} f=\left\langle\mathrm{x}^{2}(\mathrm{~T})>\cong \frac{6 \mathrm{E}_{\mathrm{R}}}{\mathrm{k} \theta_{\mathrm{D}}}\left[\frac{\mathrm{T}}{\theta_{\mathrm{D}}}\right]\right.
$$

donde ER es la energía de retroceso. En la figura 15 se muestra el gráfico del área total de absorción Mössbauer en función de la temperatura, para la muestra dopada con 3\% de Fe en un vacío de 10-2 atm. Todos los datos pueden ser consistentemente analizados con . El valor absoluto de es relativamente alto para un metal, sin embargo es típico de los óxidos de Fe [19]. No es posible un gráfico similar para las áreas de absorción versus la temperatura [20] de los dobletes cuadrupolares individuales, toda vez que hay un intercambio de una especie con otra durante las medidas.

\section{CONCLUSIONES.}

Se estudiaron muestras del sistema (La1-x Gdx)1,85 Sr0,15CuO4 dopadas con 1\% de átomos de Fe por $\mathrm{Cu}$, a la temperatura ambiente y las temperaturas de $110 \mathrm{~K}, 4,2 \mathrm{~K}$ y otras más elevadas, por espectroscopía Mössbauer, difracción de rayos X y susceptibilidad magnética. El análisis de los datos como un todo permite una interpretación de las diferentes especies de Fe en términos de las vecindades locales características de las fases $\mathrm{T}, \mathrm{T}^{*}$ y $\mathrm{T}^{\prime}$.

En lo que respecta a la simetría local, fueron necesarios cinco tipos de dobletes (especies A, B, C, D y E) para describir los espectros Mössbauer en la transición de fase $\mathrm{T}^{\circledR} \mathrm{T}^{*}{ }^{\circledR} \mathrm{T}^{\prime}$. El hecho de que los iones de Fe sean capaces de retener átomos de oxígenos extras, impide cambios drásticos de los espectros cuando se pasa de una estructura a otra, permitiendo la coexistencia de diferentes configuraciones de oxígeno.

En lo que se refiere al ordenamiento magnético, el campo magnético hiperfino desdoblado en el 57Fe, el cual es observado en estas muestras, puede no solamente reflejar un ordenamiento magnético espontáneo de la subred de $\mathrm{Cu}$, sino también un

ordenamiento local inducido por los momentos de los iones de Fe. El carácter del campo hiperfino observado depende de la configuración de oxígeno para el Fe: en el caso de las coordinaciones octaédricas y piramidales (donde existe una interac-ción magnética fuerte entre los iones de $\mathrm{Fe}$ y $\mathrm{Cu}$ ) no se espera fluctuaciones del momento magnético del Fe. Esto está de acuerdo con el espectro magnético hiperfino Mössbauer "estático" observado para las fases T y T*.

Sin embargo, en el caso de la configuración de oxígeno planar cuadrado, la magnitud de la interacción magnética Fe-Cu es más débil debido al reducido número de enlaces con los oxígenos y de esta manera los momentos del Fe son capaces de fluctuar aún cuando la subred Cu-O está magnética-mente ordenada (por debajo de $260 \mathrm{~K}$ ). La precesión es muy rápida. Sólo a 4,2K su frecuencia es comparable con el tiempo de la ventana Mössbauer llevando a un espectro de relajación magnética.

Los estudios Mössbauer in situ a altas temperaturas en la fase T' muestran un cambio irreversible de las intensidades relativas de los dobletes principales D y E. Este cambio puede ser explicado por una desorción térmica activada de oxígenos alrededor de los iones de Fe la cual tiene lugar siguiendo un proceso de difusión por encima de 170 oC.

\section{BILBLIOGRAFÍA}


[1] J.G.Bednorz and K.A.Muller,Z.PhysB 64 (1986)198.

[2] J. Bardeen, L.N.Cooper and R.J.Chieffer, Phys.Rev. 108: 1175(1957)

[3] G.Xiao, M.Z.Cieplak and C.L.Chien, Phys.Rev. B 40: 4538 (1989)

[4] Y.Tokura, H.Takagi and S.Uchida, Nature (London) 337: 345 (1989)

[5] E.Baggio-Saitovitch,I.Souza Azevedo, R.B Scorzelli, H.Saitovitch,S.F.daCunha,A.P.Guimarães,P.R.Silva and A.Y.Takeuchi, Phys.Rev. B37: 7967 (1988)

[6] P.Imbert, G.Jéhanno and J.A.Hodges, Hyperfine Interactions 55:1307 (1990)

[7] T.Shinjo, S.Nasu, T.Mizutani, K.Shintaku, N. Hosoito,K.Matsukuma and T.Takabatake, Hyperfine Interactions 55: 1379-1386 (1990)

[8] E.Baggio-Saitovitch,R.B.Scorzalli, I. Souza Azevedo and H. Micklitz, Phys. Rev. B 41: 2103 (1990)

[9] E.Baggio-Saitovitch, R.B.Scorzelli, I. Souza Azevedo, C.A. Dos Santos and F.J. Litterst, Physica C 166: 518-522 (1990)

[10] D.E. Ellis, E. Baggio Saitovitch and D.J. Lam, Physica C 198: 57 (1992)

[11] Z.Tan, M.E.Filipkowsky, J.I.Budnick, Phys.Rev. Lett. 64 (22): 2715 (1990)

[12] M.A.Márquez Jácome, Tes es de Mestrado, CBPF-Rio de Janeiro. Brasil (1993)

[13] E.Baggio-Saitovitch,M.A.Márquez,D.Rsánchez and S.G García. Physica C 235-240: 865-866 (1994)

[14] M.Müther, G.Wortmann, T.Felner and I.Novik, Physica C 209: 428 (1993)

[15] D.R.Sánchez Candela, Tese de Mestrado, CBPF-Rio de Janeiro. Brasil (1994)

[16] P. Imbert, G.Jéhanno, P.Debray, C.Garcin and J.A.Hodges, J.Phys.I France 2: 1405-1430 (1992)

[17] Dalber Sánchez,E.Baggio-Saitovitch, M.Elmassalmi and F.J.Litterst, a ser publicado en el proceeding del LACAME-CHILE (1994)

[18] N.N.Greenwood and T.C.Gibb; Mössbauer Spectroscopy, Chapman and Hall Ltd. London, (1971)

[19] G.A.Sawatzky,F.Van der Woude, and A.H.Morrish, Phys.Rev. 183: 383 (1969)

[20] D.R.Sánchez, F.J.Litterst and E.Baggio-Saitovitch a ser publicado.

San Lázaro y L, Vedado, Habana Ciudad 10400, Cuba.

\section{Ver Imágenes}

